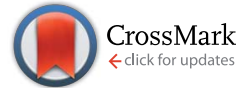

Cite this: Chem. Sci., 2017, 8, 3489

\title{
Novel bright-emission small-molecule NIR-II fluorophores for in vivo tumor imaging and image- guided surgery $\dagger$
}

\author{
Yao Sun, $\dot{t}^{\mathrm{ab}}$ Mingmin Ding, $\dot{t}^{\mathrm{a}}$ Xiaodong Zeng, $\dot{t}^{\mathrm{a}}$ Yuling Xiao, $\dot{t}^{\mathrm{a}}$ Huaping $\mathrm{Wu},{ }^{a}$ \\ Hui Zhou, ${ }^{a}$ Bingbing Ding, ${ }^{a}$ Chunrong Qu, ${ }^{a}$ Wei Hou, ${ }^{a}$ AGA Er-bu, ${ }^{d}$ Yejun Zhang, ${ }^{e}$ \\ Zhen Cheng ${ }^{c}$ and Xuechuan Hong*ad
}

\begin{abstract}
Though high brightness and biocompatible small NIR-II dyes are highly desirable in clinical or translational cancer research, their fluorescent cores are relatively limited and their synthetic processes are somewhat complicated. Herein, we have explored the design and synthesis of novel NIR-II fluorescent materials (H1) without tedious chromatographic isolation with improved fluorescence performance (QY $\approx 2 \%$ ) by introducing 2-amino 9,9-dialkyl-substituted fluorene as a donor into the backbone. Several types of water-soluble and biocompatible NIR-II probes: SXH, SDH, and H1 NPs were constructed via different chemical strategies based on $\mathrm{H} 1$, and then their potential to be used in in vivo tumor imaging and image-guided surgery in the NIR-II region was explored. High levels of uptake were obtained for both passive and active tumor targeting probes SXH and SDH. Furthermore, high resolution imaging of blood vessels on tumors and the whole body of living mice using H1 NPs for the first time has demonstrated precise NIR-II image-guided sentinel lymph node (SLN) surgery.
\end{abstract}

Received 18th January 2017

Accepted 18th February 2017

DOI: $10.1039 / \mathrm{c} 7 \mathrm{sc} 00251 \mathrm{c}$

rsc.li/chemical-science inorganic materials such as small molecules, ${ }^{5-7}$ conjugated polymers, ${ }^{8}$ carbon nanotubes, ${ }^{\mathbf{9}, 10}$ quantum dots (QDs), ${ }^{\mathbf{1 1 - 1 3}}$ and rare earth nanoparticles, ${ }^{\mathbf{1 4 , 1 5}}$ have been actively employed for NIR-II fluorescence imaging: ${ }^{16}$ However, reports of NIR-II fluorophores are still scarce and the small-molecule fluorescent cores are relatively limited compared with their NIR-I counterparts. ${ }^{6}$ Hence, it prompts us to expand the library of smallmolecule NIR-II fluorophores, which will significantly promote the widespread use of NIR-II imaging modality.

Several types of small-molecule NIR-II dyes with favorable excretion pharmacokinetics have been reported, in which the fluorophore units are generally composed of aromatic conjugate units based on a donor-acceptor-donor (D-A-D) structure with a benzobisthiadiazole (BBTD) core., ${ }^{5,6}$ Among them, a small-molecule probe Q4 was selected as a scaffold for the facile construction of NIR-II agent SCH1100 for targeted prostate cancer imaging. ${ }^{6,17}$ However, the complexity and multiple synthetic steps with low yields, tedious chromatographic isolation and the weak brightness of Q4 heavily hinder the wide application of such a promising agent in preclinical and clinical studies. Hence, many efforts should be made to simplify the synthetic strategy and optimize the brightness of smallmolecule NIR-II fluorophores.

Herein, we report a novel small-molecule NIR-II dye H1 with an improved synthetic protocol and fluorescence characteristics. At longer fluorescence emission wavelengths in the NIR-II region, the increased bandgap of molecular fluorophores generally gives way to reduce interactions between the 
conjugated backbone and other molecules, causing a high fluorescence quantum yield (QY). ${ }^{\mathbf{1 8 , 1 9 2 0}}$ Therefore, in this work, $\mathrm{R}_{1}$ substituent groups on the $\mathrm{sp}^{3}$ carbon of the fluorene group are out-of-plane of the $\pi$-conjugated system and thus prevent intermolecular stacking that leads to fluorescence quenching. Meanwhile, newly introduced 2-amino 9,9-dialkyl-substituted fluorene moieties distort the BBTD backbone and thus effectively tune the electrostatic potential distribution and the bandgap to the desired range. Moreover, the fluorene moieties act as both the electron donor and protecting groups with the benefits of a compact molecular structure and shielding the backbone from aggregation (Fig. 1). Finally, three types of NIR-II probes (SXH, SDH, and H1 NPs) have been facilely prepared according to the $\mathbf{H 1}$ scaffold, and demonstrated different biomedical applications such as passive/active tumor targeted imaging, high resolution imaging of blood vessels on tumors and the whole body, and image-guided sentinel lymph node surgery in the NIR-II imaging region. The novel organic fluorescent compound $\mathbf{H 1}$ provides unprecedented opportunities for the construction of a variety of NIR-II probes for in vivo molecular imaging.

\section{Results and discussion}

As shown in Fig. 1, compound $\mathbf{H 1}$ was synthesized by a convergent route in 15\% overall yield over 6 steps from commercially available starting materials without tedious chromatographic isolation (see ESI $\dagger$ ). All compounds were characterized by NMR and ESI-MS, and also exhibited good solubility in common organic solvents such as $\mathrm{CH}_{2} \mathrm{Cl}_{2}$ and THF (see ESI $\dagger$ ). By modulating the non-resonant side chains of the carboxylic acid groups, the final NIR-II fluorophores could be systematically altered to tune the hydrophobicity, polarity and efficient conjugation of bio-targets. The UV-vis-NIR absorption band of $\mathrm{H1}$ was at $600-1000 \mathrm{~nm}$ (in $\mathrm{CH}_{2} \mathrm{Cl}_{2}$ ) due to the formation of a strong charge-transfer structure between the D-A-D units (Fig. 2a). Meanwhile, the fluorescence emission spectrum of H1 was obtained and demonstrated a peak emission wavelength at $\sim 1100 \mathrm{~nm}$ (Fig. 2a). The results indicated that the brightness of the fluorescence signals of $\mathbf{H 1}$ was superior to that of Q4 (Fig. 2b). Furthermore, the NIR-II signals of $\mathbf{H 1}$ were investigated under various LP filters (900-1400 nm) and no signals were observed with the $1300 \mathrm{~nm}$ and $1400 \mathrm{~nm}$ filters (Fig. 2c). H1 has exhibited high photo-stability compared to IR-26, with negligible decay under continuous excitation for $1 \mathrm{~h}$ (Fig. 2d).

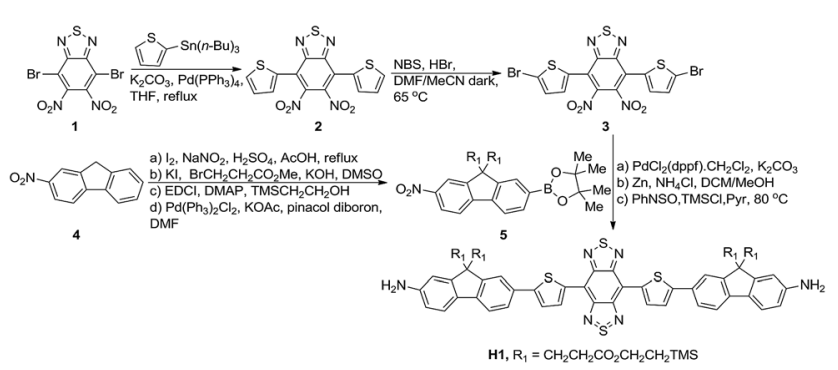

Fig. 1 Facile synthesis of compound $\mathrm{H} 1$. a)

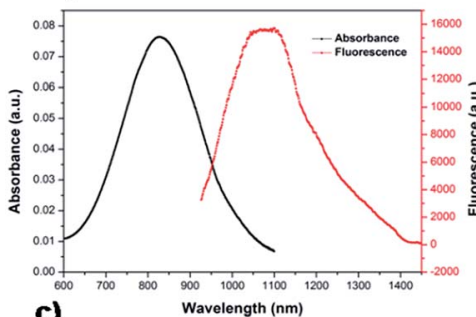

c) b)

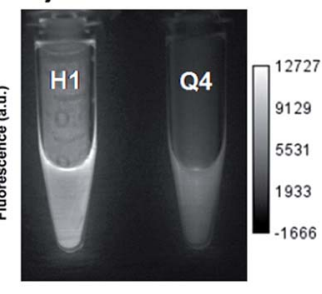

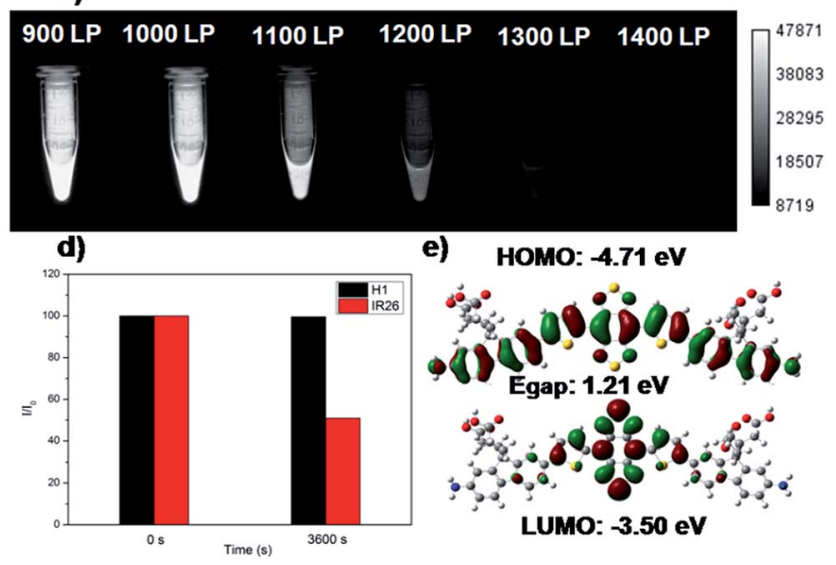

Fig. 2 (a) UV absorbance of $\mathrm{H} 1$ and NIR-II fluorescence emission of $\mathrm{H} 1$ with a peak at $\sim 1100 \mathrm{~nm}$ under an $808 \mathrm{~nm}$ excitation laser (exposure time: $10 \mathrm{~ms}$ ). (b) Comparison of NIR-II signals of $\mathrm{H} 1$ and $\mathrm{Q} 4$ under an $808 \mathrm{~nm}$ excitation laser (exposure time: $20 \mathrm{~ms}$ ). (c) Comparison of NIR-II signals of $\mathrm{H} 1$ under various long-pass (LP) filters (900-1400 nm). (d) Comparison of the photo-stability of $\mathrm{H} 1$ and IR-26 in dichloromethane under continuous $808 \mathrm{~nm}$ excitation for $1 \mathrm{~h}$. (e) HOMO and LUMO orbital surfaces of $\mathrm{H} 1$ using the DFT B3LYP/6$31 \mathrm{G}(\mathrm{d}) \mathrm{scrf}=\left(\mathrm{cpcm}\right.$, solvent $\left.=\mathrm{CH}_{2} \mathrm{Cl}_{2}\right)$ method. $E_{\text {gap }}=E_{\mathrm{LUMO}}-$ Eномо.

The calculated HOMO and LUMO orbital surfaces of $\mathbf{H 1}$ have shown a larger band gap compared to that of $\mathbf{Q 4}$, leading to a higher performing fluorophore (Fig. 2e and Table S1†). The QY of $\mathbf{H 1}$ was $\sim 2.0 \%$ under $785 \mathrm{~nm}$ excitation (in $\mathrm{CH}_{2} \mathrm{Cl}_{2}$, measured against an IR-26 reference with a nominal quantum yield of $0.5 \%$, Fig. S1 $\dagger$ ). All these data demonstrated $\mathbf{H 1}$ could be a promising NIR-II dye, suitable for further NIR-II imaging applications.

SXH was easily prepared through conjugation of four carboxylic acid groups of $\mathbf{H 1}$ with PEG $_{1000}$ chains (Fig. 3a and ESI $\dagger$ ). SXH was purified using HPLC and characterized using MALDI-TOF-MS (see ESI $\dagger$ ). SXH exhibited high aqueous solubility and the fluorescence emission spectrum of SXH demonstrated a similar emission wavelength at $\sim 1100 \mathrm{~nm}$ to that of $\mathbf{H 1}$ (Fig. 3b). The results from a cytotoxicity study further indicated the high viability of U87MG and L929 cells after $24 \mathrm{~h}$ of incubation with different doses of SXH $(2,4,6$, and $8 \mu \mathrm{M})$, demonstrating the high biocompatibility of SXH (Fig. 3c). Excretion kinetics were investigated by intravenous injection of $100 \mu \mathrm{g}$ of SXH into U87MG tumor-bearing nude mice $(n=3)$ for glioblastoma (GBM) imaging and collecting urine during the course of $24 \mathrm{~h}$ post-injection (P.I.). Glioblastoma, the most common primary brain tumor in adults, is usually rapidly fatal. ${ }^{21}$ The care 

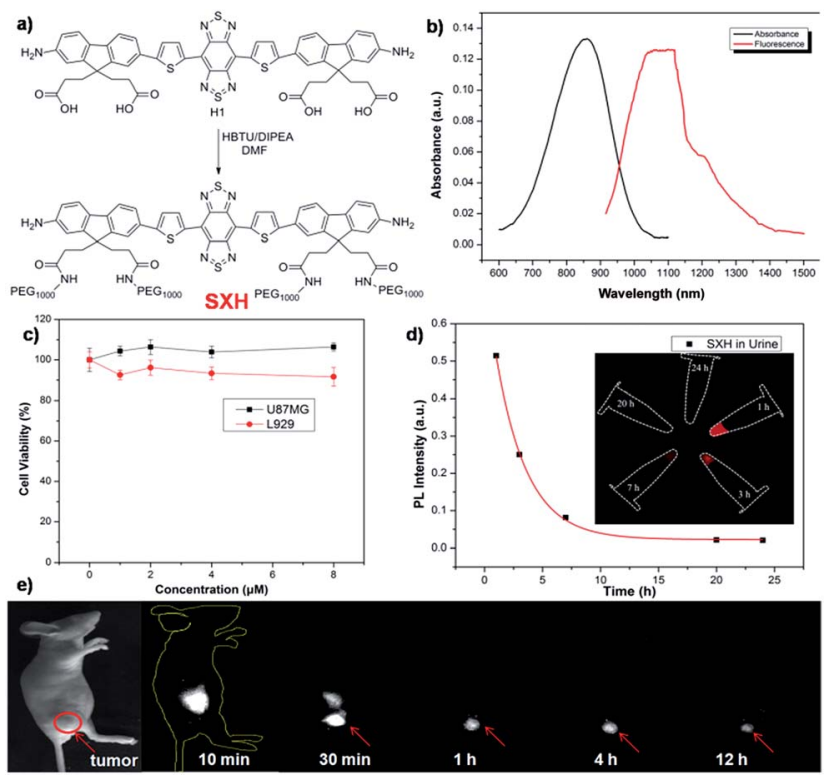

Fig. 3 (a) A schematic design of SXH showing four carboxylic acid groups of $\mathrm{H} 1$ conjugated with $\mathrm{PEG}_{1000}$ chains. (b) UV absorbance of SXH and NIR-II fluorescence emission of SXH with a peak at $1100 \mathrm{~nm}$ under an $808 \mathrm{~nm}$ excitation laser (solvents: water, exposure time: 10 $\mathrm{ms})$. (c) Cellular toxicity of SXH with different doses $(2,4,6$, and $8 \mu \mathrm{M})$ in U87MG and L920 cells. (d) SXH agglomerated cumulative urine excretion curve during $24 \mathrm{~h}$ post-injection. (e) Non-specific targeting imaging of the U87MG tumor based on SXH under an $808 \mathrm{~nm}$ excitation (1000 LP and $200 \mathrm{~ms}$ ).

for patients with a newly diagnosed glioblastoma entails surgical resection and concurrent radiation therapy (RT) and chemotherapy. Pharmacokinetics of SXH demonstrated rapid urine excretion, with $\sim 90 \%$ of SXH removal through the renal system within the first few hours of the $24 \mathrm{~h}$ post-injection (Fig. 3d and Fig. S2 $\dagger$ ). Finally, U87MG tumor-bearing nude mice $(n=3)$ were injected with $100 \mu \mathrm{g}$ of SXH and non-invasive NIR-II fluorescence imaging of the glioblastoma tumor was conducted at particular time points. After $30 \mathrm{~min}$ post-injection, the tumor was clearly visible with a T/NT ratio of $\sim 4$ and showed passive uptake at all time points due to the non-specific diffusion and accumulation of SXH (Fig. 3e). Ex vivo biodistribution studies were further performed at $24 \mathrm{~h}$ post-injection of the probe to evaluate the distribution of SXH in major organs (Fig. S3†). It was found that SXH mainly accumulated in the kidneys, suggesting that the clearance route of SXH was through the renal system. In addition, a high level of accumulation was also observed in the tumor, indicating that SXH can passively target tumors and be used for future cancer theranostic applications (Fig. $\mathrm{S} 3 \dagger$ ).

Although PEGylation of $\mathbf{H 1}$ provided a rapidly excreted, versatile contrast agent capable of passive tumor uptake, H1 could provide more tumor-specific targeting by linking to a molecular imaging ligand. We next demonstrated the application of $\mathbf{H 1}$ for receptor-targeted glioma imaging. Integrin $\alpha_{\mathrm{V}} \beta_{3}$ has high expression levels in several malignant diseases including glioblastoma and are established biomarkers for metastatic diseases. ${ }^{22}$ The integrin targeting peptide RGD (arginine-glycine-aspartic acid) has shown promising results for non-invasive molecular imaging of integrin $\alpha_{V} \beta_{3}$ expression in the NIR-I region. ${ }^{23}$ Considering the advantages of NIR-II imaging, a novel integrin $\alpha_{\mathrm{V}} \beta_{3}$-targeted NIR-II fluorophore, SDH, was developed and explored to investigate its imaging properties in vivo. SDH was prepared through conjugation of $\mathbf{H 1}$ with a mono-c(RGDfk) targeting peptide (Fig. 4a), and then purified by HPLC and characterized by MALDI-TOF [calcd for $\mathrm{C}_{72} \mathrm{H}_{79} \mathrm{~N}_{15} \mathrm{O}_{14} \mathrm{~S}_{4}$ : 1589.481, found: $m / z$ 1589.669]. The fluorescence emission spectrum of SDH demonstrates an emission wavelength at $\sim 1050 \mathrm{~nm}$ (Fig. 4b). The cell toxicity study also indicated the high biocompatibility of SDH in vitro (U87MG and L929 cells after $24 \mathrm{~h}$ incubation with $2,4,6$, and $8 \mu \mathrm{M}$ doses of SDH, Fig. S4 $\dagger$ ). These results demonstrated that SDH, as a promising and biocompatible NIR-II fluorescent probe, is suitable for tumor targeting imaging.

SDH was then intravenously injected $(100 \mu \mathrm{g})$ into U87MG tumor-bearing mice ( $n=3$ per group). From NIR-II imaging data, the U87MG tumor could be clearly visualized from the surrounding background tissue during 24-72 h post-injection (P.I.) (Fig. 4d, 1000LP, $200 \mathrm{~ms}$ ), and the tumor uptake reached a maximum at $48 \mathrm{~h}$. The specificity of SDH for integrin $\alpha_{V} \beta_{3}$ was confirmed by the blocking experiment. The tumors fluorescence signals were successfully reduced at all time points after coinjection of RGD peptide $(500 \mu g)$ with SDH for NIR-II imaging (Fig. 4d). An ex vivo biodistribution study indicated that high accumulation was observed in the liver and kidneys, which suggested that the clearance routes of SDH were through both hepatobiliary and renal systems (Fig. S5†). In addition, the uptake of SDH in tumors was far beyond that of other normal organs and no uptake could be observed in the blocking group,

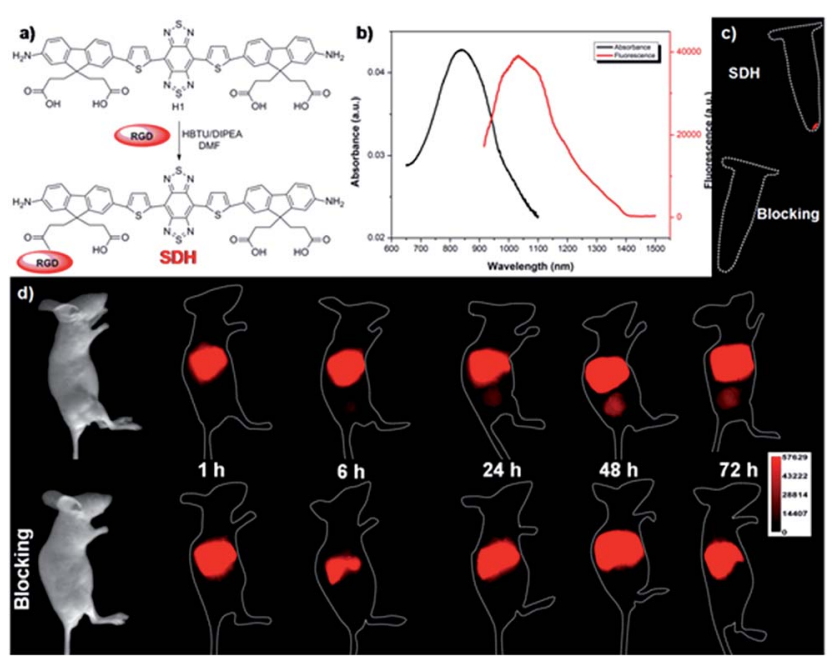

Fig. 4 (a) A schematic of SDH showing one of the carboxylic acid groups of $\mathrm{H} 1$ conjugated with targeted ligand RGD peptide. (b) UV absorbance and NIR-II fluorescence emission of SDH. (c) NIR-II signals of U87MG cell labelling by SDH and SDH + excess RGD as a blocking agent (block group) under $808 \mathrm{~nm}$ excitation (1000LP and $100 \mathrm{~ms}$ ). (d) NIR-II images of U87MG tumor mice $(n=3)$ at different time points ( 1 , $6,24,48$, and $72 \mathrm{~h}$ ) after tail vein injection of SDH with or without the blocking agent RGD $(500 \mu \mathrm{g})$ under $808 \mathrm{~nm}$ excitation (1000LP and $200 \mathrm{~ms})$. 
which further confirmed the good integrin $\alpha_{V} \beta_{3}$-targeted ability and specificity of SDH (Fig. S5 and S6†). Hence, the excellent translation ability of SDH represents a highly promising fluorescent probe for non-invasive monitoring of early stage glioblastoma in the NIR-II region.

An emerging fluorescence imaging application of NIR fluorophores, such as indocyanine green (ICG), is currently undergoing clinical trials in detecting sentinel lymph nodes (SLNs) for surgical resection. ${ }^{24}$ Selectively removing sentinel lymph nodes alleviates lymphedema and other ailments that would be caused by total lymph node removal, which is performed to prevent cancer metastasis. Recent advances in NIR-I fluorescence molecular imaging (FMI) for intraoperative image-guided cancer resection have introduced new frontiers for FMI-based therapeutic interventions in preclinical research and clinical applications. The inherent advantages of this novel technology compared to bright light surgery, such as its high sensitivity, high superficial resolution, low cost, and real-time imaging capacity, have stimulated the development of fluorescent probes with different molecular features. ${ }^{25-33}$ To demonstrate the feasibility of $\mathbf{H 1}$ for SLN surgery, $\mathbf{H 1}$ was encapsulated into a PEGylated surfactant, DSPE-mPEG ${ }_{5000}$, to prepare watersoluble and biocompatible NIR-II nanoprobes, H1 NPs (Fig. 5a). The prepared H1 NPs showed high monodispersity and homogeneity with an average particle size of $\sim 70.0 \mathrm{~nm}$ by transmission electron microscopy (TEM, Fig. 5b) and a hydrodynamic diameter of $\sim 80.0 \mathrm{~nm}$ as determined by dynamic light scattering (DLS, Fig. S7†). The fluorescence emission wavelength of H1 NPs was $\sim 1100 \mathrm{~nm}$ (Fig. S8†). The result of the cytotoxicity study indicated the high biocompatibility of H1 NPs

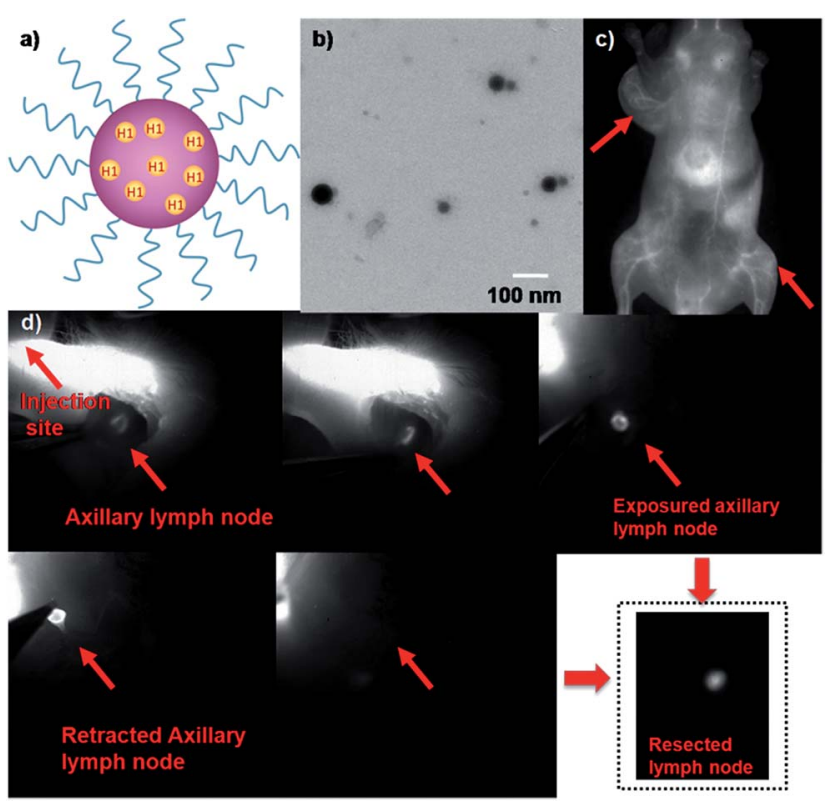

Fig. 5 (a) A schematic of H1 NPs showing the $\mathrm{H} 1$ core and a hydrophilic PEG shell. (b) The TEM image of H1 NPs. (c) The NIR-II image of the blood vessels of the whole body and U87MG tumors after tail vein injection of H1 NPs under $808 \mathrm{~nm}$ excitation, $1000 \mathrm{LP}$ and $100 \mathrm{~ms}$; red arrows indicate the tumor. (d) NIR-II imaging-guided sentinel lymph node surgery on the C57BL/6J model (1000LP and $200 \mathrm{~ms}$ ).
(Fig. S9†). The amount of $\mathbf{H 1}$ encapsulated in the liposomes was measured by a UV/VIS spectrophotometer at $874 \mathrm{~nm}$ (ESI $\dagger$ ). The dye encapsulation efficiency of H1 NPs was $79.8 \pm 0.6 \%(n=3)$.

The U87MG tumor-bearing nude mice $(n=3)$ were injected with $150 \mu \mathrm{L}$ of $\mathbf{H 1}$ NPs. Immediately after injection, the superior imaging of blood vessels of the whole body and tumor could be clearly observed from the surrounding background tissue using NIR-II imaging (Fig. 5c). Based on this promising result, we applied H1 NPs for lymph node imaging and image-guided surgery on the C57BL/6J model (Fig. 5d). C57BL/6J mice were injected with $100 \mu \mathrm{L}$ of $\mathbf{H 1}$ NPs, with the help of NIR-II imaging, a SLN was successfully determined, even covered with soft tissue. After the SLN was exposed, it was then resected thoroughly. More importantly, the border of the SLN was easily distinguished, avoiding unnecessary damage of surrounding tissue such as nerves, vessels and tendons.

\section{Conclusions}

In summary, we have developed a newly designed and facilely prepared NIR-II fluorophore $\mathbf{H 1}$ with improved fluorescence by introducing 2-amino 9,9-dialkyl-substituted fluorene as a donor into the backbone. Based on this H1 scaffold, three types of NIRII imaging probe, SXH, SDH and H1 NPs, have been prepared and allow for integrin $\alpha_{V} \beta_{3}$-targeted glioma imaging. To the best of our knowledge, this is the first time that a NIR-II molecular fluorophore has been shown to delineate tumours from surrounding normal tissue. Various biomedical applications such as high resolution imaging of blood vessels on tumours and the whole body of living mice using H1 NPs were also performed through a passive targeted probe. With the help of NIR-II imaging, an SLN was successfully determined and resected thoroughly. Future work will focus on performing intraoperative image-guided cancer surgery in orthotopic models and specialized pharmacokinetic studies with the eventual goal of initiating clinical trials with a NIR-II smallmolecule fluorophore.

\section{Acknowledgements}

This work was partially supported by NSFC (81573383, 21390402, 81301268), NSFHP (2014CFB704), IS\&TCPC (2015DFA30440, 2014CFB30020), the Major Project of Technology Innovation Program of Hubei Province (2016ACA126), the Applied Basic Research Programs of Scientific and Technologic Council of Suzhou (SYG201521), the Natural Science Foundation of Jiangsu Province (BK20160387), Beijing Nova Plan Z (131107000413063), and the Fundamental Research Funds for the Central Universities.

\section{Notes and references}

1 G. S. Hong, A. L. Antaris and H. J. Dai, Nat. Biomed. Eng., 2017, 1, 0010.

2 G. S. Hong, J. C. Lee, J. T. Robinson, U. Raaz, L. M. Xie, N. F. Huang, J. P. Cooke and H. J. Dai, Nat. Med., 2012, 18, 1841-1846. 
3 G. S. Hong, S. Diao, A. L. Antaris and H. J. Dai, Chem. Rev., 2015, 115, 10816-10906.

4 C. Y. Li, Y. J. Zhang, M. Wang, Y. Zhang, G. C. Chen, L. Li, D. M. Wu and Q. B. Wang, Biomaterials, 2014, 35, 393-400.

5 A. L. Antaris, H. Chen, K. Cheng, Y. Sun, G. S. Hong, C. R. Qu, S. DIao, Z. X. Deng, X. M. Hu, B. Zhang, X. D. Zhang, O. K. Yaghi, Z. R. Alamparambil, X. C. Hong, Z. Cheng and H. J. Dai, Nat. Mater., 2016, 15, 235-242.

6 Y. Sun, C. Q. Rong, H. Chen, M. M. He, C. Tang, K. Q. Shou, S. Hong, M. Yang, Y. X. Jiang, B. B. Ding, Y. L. Xiao, L. Xing, X. C. Hong and Z. Cheng, Chem. Sci., 2016, 7, 6203-6207.

7 W. H. Zhu, Sci. China: Chem., 2016, 59, 203-204.

8 G. S. Hong, Y. P. Zhou, A. L. Antaris, S. Diao, D. Wu, K. Cheng, X. D. Zhang, C. X. Chen, B. Liu, Y. H. He, J. Z. Wu, J. Yuan, B. Zhang, Z. M. Tao, C. Fukunaga and H. J. Dai, Nat. Commun., 2014, 5, 4206.

9 G. S. Hong, S. Diao, J. L. Chang, A. L. Antaris, C. X. Chen, B. Zhang, S. Zhao, D. N. Atochin, P. L. Huang, K. I. Andreasson, C. J. Kuo and H. J. Dai, Nat. Photonics, 2014, 8, 723-730.

10 S. Diao, J. L. Blackburn, G. S. Hong, A. L. Antaris, J. L. Chang, J. Z. Wu, B. Zhang, K. Cheng, C. J. Kuo and H. J. Dai, Angew. Chem., Int. Ed., 2015, 54, 14758-14762.

11 Y. Zhang, G. S. Hong, Y. J. Zhang, G. C. Chen, F. Li, H. J. Dai and Q. B. Wang, ACS Nano, 2012, 6, 3695-3702.

12 G. S. Hong, J. T. Robinson, Y. J. Zhang, S. Diao, A. L. Antaris, Q. B. Wang and H. J. Dai, Angew. Chem., Int. Ed., 2012, 51, 9818-9821.

13 G. C. Chen, F. Tian, C. Y. Li, Y. J. Zhang, Z. Weng, Y. Zhang and Q. B. Wang, Biomaterials, 2015, 53, 265-273.

14 D. J. Naczynski, M. C. Tian, M. Zevon, B. Wall, J. Kohl, A. Kulesa, S. Chen, C. M. Roth, R. E. Riman and P. V. Moqhe, Nat. Commun., 2013, 4, 2199.

15 X. Y. Jiang, C. Cao, W. Feng and F. Y. Li, J. Mater. Chem. B, 2016, 4, 87-95.

16 X. N. Dang, L. Gu, J. F. Qi, S. Correa, G. R. Zhang, A. M. Belcher and P. T. Hammond, Proc. Natl. Acad. Sci. U. S. A., 2016, 113, 5179-5184.

17 Y. Sun, X. Ma, Z. Zhang, Z. Y. Sun, M. Loft, B. B. Ding, C. H. Liu, L. Y. Xu, M. Yang, Y. X. Jiang, J. F. Liu,
Y. L. Xiao, Z. Cheng and X. C. Hong, Bioconjugate Chem., 2016, 27, 1857-1864.

18 G. Qian, Z. Zhong, M. Luo, D. B. Yu, Z. Q. Zhang, Z. Y. Wang and D. G. Ma, Adv. Mater., 2009, 21, 111-116.

19 G. Qian, J. P. Gao and Z. Y. Wang, Chem. Commun., 2012, 48, 6426-6428.

20 X. D. Zhang, H. Wang, A. L. Antaris, L. I. Li, S. Diao, R. Ma, A. Nguyen, G. S. Hong, Z. R. Ma, J. Wang, S. J. Zhu, J. M. Castellano, T. Wyss-Coray, Y. Y. Liang, J. Luo and H. J. Dai, Adv. Mater., 2016, 28, 6872-6879.

21 R. Stupp, et al., N. Engl. J. Med., 2005, 352, 987-996.

22 M. Pfaff, K. Tangemann, B. Muller, M. Gurrath, G. Muller, H. Kessler, R. Timpl and J. Engel, J. Biol. Chem., 1994, 269, 20233-20238.

23 Y. Sun, X. Ma, K. Cheng, B. Wu, J. Duan, H. Chen, L. Bu, R. Zhang, X. Hu, Z. Deng, L. Xing, X. Hong and Z. Cheng, Angew. Chem., Int. Ed., 2015, 54, 5981-5984.

24 E. Tanaka, H. S. Choi, H. Fujii, M. G. Bawendi and J. V. Frangioni, Ann. Surg. Oncol., 2006, 13, 1671-1681.

25 M. Momiyama, Y. Hiroshima, A. Suetsugu, Y. Tome, S. Mii, S. Yano, M. Bouvet, T. Chishima, I. Endo and R. M. Hoffman, Anticancer Res., 2013, 33, 107-112.

26 R. M. Hoffman, Invest. New Drugs, 1999, 17, 343-359.

27 R. M. Hoffman, Nat. Rev. Cancer, 2015, 15, 451-452.

28 R. M. Hoffman and M. Yang, Nat. Protoc., 2006, 1, 14291438.

29 C. A. Metildi, S. Kaushal, C. R. Hardamon, C. S. Snyder, M. Pu, K. S. Messer, M. A. Talamini, R. M. Hoffman and M. Bouvet, J. Am. Coll. Surg., 2012, 215, 126-135.

30 C. A. Metildi, S. Kaushal, M. Pu, K. A. Messer, G. A. Luiken, A. R. Moossa, R. M. Hoffman and M. Bouvet, Ann. Surg. Oncol., 2014, 21, 1405-1411.

31 C. A. Metildi, S. Kaushal, C. S. Snyder, R. M. Hoffman and M. Bouvet, J. Surg. Res., 2013, 179, 87-93.

32 T. Murakami, Y. Hiroshima, Y. Zhang, M. Bouvet, T. Chishima, K. Tanaka, I. Endo and R. M. Hoffman, J. Surg. Oncol., 2015, 112, 119-124.

33 Y. Hiroshima, A. Maawy, Y. Zhang, S. Sato, T. Murakami, M. Yamamoto, F. Uehara, S. Miwa, S. Yano, M. Momiyama, T. Chishima, K. Tanaka, M. Bouvet, I. Endo and R. M. Hoffman, PLoS One, 2014, 9, e99977. 